\title{
CASE OF SUB-MAXILLARY TUMOR-TRACHEOTOMY.
}

BY B. R. RAPHAEL, M.D., N. YORK.

JohN Ulabroph, ætat. 21, milkman, born in New York, was admitted to the New York Hospital, May 23d, 1840, with a swelling under the lower jaw, which very much impeded his respiration. As far as could be ascertained, the first symptom he had of the disease was enlargement of the tonsils; one of these was removed last January. From that time a hard, but not very tender swelling seemed to spread, until it reached its present size. It occupied the whole sub-maxillary region from one ear to the other, and reached down to the os hyoides, which was depressed by it. In the buccal cavity it had encroached very much, so that the tongue was protruded and raised up. It was firmly fixed to the jaw and os hyoides. The patient could not open his mouth more than one third of an inch ; deglutition was very difficult, and respiration exceedingly embarrassed. His face was very red, approaching round the lips to purple; countenance expressive of the greatest anxiety. The tumor had only affected his breathing for four days previous to his admission, and as he had not slept during the whole time, he was very drowsy. He could not endure the recumbent posture for a moment.

May 24th. Slept none last night; breathing even more difficult than yesterday, each act of respiration accompanied with a loud moan. In consultation, laryngotomy was unanimously advised. It was performed by Dr. A. C. Post, at 2 P. M., in the crico-thyroid space. As soon as a free opening was made, the air rushed in with a hissing noise, and with great relief to the patient. The edges of the wound were drawn apart with threads passed through the skin, and this tied behind the neck. A few minutes after the operation he had a paroxysm of coughing, and threw out of the opening a large quantity of mucus. Soon after this he fell asleep, and during the afternoon was comfortable; occasionally, however, throwing up a quantity of bloody mucus. He slept soundly until 4 o'clock next morning.

25 th. Countenance improved; breathing easy, about 20 per minute; feels quite comfortable; bowels opened in the evening by an enema.

26 th. Slept very well; breathing very easy; nearly every hour since the operation he had a paroxysm of coughing, in which he would throw up blood mixed with mucus; after the paroxysm he would be comfortable. The tumor having previously been moistened, was touched to-day with the solid nitrate of silver.

27th. Doing extremely well; appetite very good. Is allowed milk and soft custard, which he swallows easily. A canula was introduced into the opening in the larynx; at first it created some irritation, which soon afterwards subsided. It remained in until 12 at night, when it became clogged with mucus and was removed.

28th. Canula introduced again early in the morning, and kept in until 3 P. M., when it was removed, cleansed, and again re-applied-kept in until 10 P. M.

29th. Canula introduced again.

30th. The tumor coated over with tinct. iodine; canula still remaining. 
June 8th. Keeps the instrument in for $\mathbf{2 4}$ bours without any difficulty.

10th. After removing the instrument to be cleansed this morning: the granulations seemed to swell and close the opening, and nearly stopped his breathing. It was instantly put back again, and he breathed with ease.

15th. The tumor under the jaw has diminished very much since last report, and has become softer. It would probably present no difficulty to his breathing now, but there exists in the back part of his mouth a large projecting tumor which seems entirely to close the fauces. He opens his mouth so little yet, that the exact nature of the tumor cannot be ascertained.

19th. To-day he hawked up from the back part of his mouth a very large, bad-smelling slough, of a grayish color, and as large as a goodsized oyster. After the discharge of this slough he found he could breathe more easily through his mouth. It was not accompanied or preceded by any discharge of pus other than the usual muco-purulent discharge. On examination of the fauces, a small pendulous tumor can be seen on the base of the tongue, which has its origin on the right side, anterior to the tonsil. Posterior to this, and where the tonsil should be, is a cavity apparently the situation from which the slough proceeded. The external swelling has almost entirely subsided.

30th. Since last date he has improved rapidly. By closing the orifice in the trachea, he is able to speak, whistle, blow his nose, and breathe freely. His general health is pretty good. Since last report he has been out of bed most of the time, and several times out of doors. The sore is contracting. Granulations touched with caustic and dressed with simple salve.

July 1st. The tube was removed to-day, and the orifice allowed to commence healing.

3d. The orifice has closed, and he breathes freely through the natural passage.

9th. There is some slight increase of the swelling. Tinct. iodine applied over its whole external surface.

16th. The swelling has continued to increase very rapidly. It is principally confined to the parts immediately under the tongue and the anterior part of the jaw. It is very hard, but not painful. It does not yet affect respiration. On the 13th a dozen leeches were applied, but with no marked benefit. Ordered tobacco poultice.

20th. Tumor has not increased a great deal. He feels a throbbing pain in it to-day for the first time, and says he had occasional rigors all day yesterday. All kinds of local applications were made without benefit; leeches, blisters, ung. hyd. pot., \&c.

Aug. 4th. After a consultation, two deep incisions were made on the left side of the jaw, and one on the right side, penetrating through the genio-hyoid fascia. These incisions bled very freely, and it w'as necessary to apply nitrate of silver to their surface; lint wet with colll water was then applied, and afterwards a large poultice. These measures were attended with but little benefit.

9th. The tumor has continued to increase so much, that there is now 
the greatest oppression of the respiration, and at times suffocation is imminent. Dr. Post opened the larynx in the same situation as before, and with immediate relief to his breathing. The disease still continued steadily to advance, pushing upwards and protruding the tongue, which was itself very much swollen. His bowels were kept open by laxatives; his diet was principally milk.

17th. Two incisions were made, one into each side of the tongue; they bled freely. This gave temporary relief. The patient breathes very easily through the tube. A bread and milk poultice was applied over the tongue and mouth.

23d. His condition is somewhat improved: the swelling has somewhat diminished, especially under the jaw. The incisions are nearly healed, and he is able to walk about the ward. He complains of severe smarting in that part of bis tongue which is protruded. This was relieved by the application of linseed oil and lime water.

Sept. 16th. Last night a considerable hemorrhage took place from the mouth, about $\xi$ viij. of blood were lost, by which he was much weakened. On introducing the finger along the side of the tongue, it was imbued with a most disgusting smell, which could scarcely be washed off. He has now become much emaciated and feeble; he has also a severe catarrh, and the tube is almost constantly obstructed. His appetite continues very good, and he is able to be up occasionally for a time. The edge and lower surface of the tongue has become deeply ulcerated by the pressure of the teeth.

23d. Was attacked with severe diarrhœa, which weakened him very much, but was checked without much difficulty. His appetite lately has been enormous.

Oct. 2d. Without any change in the symptoms, he was found by the patients dead in his bed. For some days past he has appeared somewhat better; the tongue had diminished a little, but on the lower part the progress of ulceration and sloughing had nearly separated it.

Post-mortem Examination.-Emaciation extreme; the whole of the tongue back to its root was greatly enlarged, and of a cartilaginous hardness. The under surface of the tongue was much destroyed by ulceration and sloughing. All the surrounding parts were involved in an almost uniform enlargement and induration. The swelling was greatest on the right side, and had pushed the epiglottis backwards and to the left. The jaw against which this tumor had so long laid in contact, was very much thinned by absorption; and the teeth could not be brought together after all the soft parts had been removed, from the change in the ligaments and glenoid articulation. The edge of the opening in the trachea was slightly ossified, and the mucous membrane for an inch below the orifice was ulcerated, exactly of the shape and size of the side of the tube he had worn. There were no traces of inflammation of the air passages. High inflanmatory redness of the cæcum, colon, and lower part of the small intestines. - Head not examined.-N. Y. Jour. of Med. and Surg. 